centres taking part in the 2015 audit, steroid exposure rates fell from $30 \%$ to $23.8 \%(p=0.003)$ and steroid excess from $13.7 \%$ to $11.5 \%$ ( $\mathrm{p}=\mathrm{NS}$ ). Steroid exposure and excess rates for sites that had not been part of the previous audit were significantly higher $(31.0 \%$ excess, $17.1 \%$ exposure, $\mathrm{p}=0.0001$ for both). There were no significant differences in important baseline characteristics of 2 groups of sites. Logistic regression analysis revealed independent predictors of reduced risk of steroid excess, after correction for disease severity. For CD these included treatment with anti-TNF therapy $(p=0.04)$, treatment in a centre with regular IBD multidisciplinary team (MDT) meetings $(p=0.01)$ and treatment in an original 2015 centre $(p=0.02)$. For UC treatment in a 2015 centre was also significant predictor of protection $(p=0.04)$ and treatment with thiopurine monotherapy a predictor of risk of excess $(p=0.01)$; usage of anti-TNF therapy in UC did not reach significance for protection from excess.

Conclusions Changes in biologic access in the UK have resulted in only modest changes in prescribing behaviour and have not yet impacted significantly on excess steroid exposure in UC, unlike in CD. Participation in an audit cycle of steroid usage was associated with a meaningful reduction in steroid excess. These data support the concept that steroid excess could be used as a key performance indicator in IBD and physicians should be engaged in this process.

\section{ADTU-04 FAECAL CALPROTECTIN IN PSC-IBD: A NOVEL MARKER OF CHOLANGITIS}

Polychronis Pavlidis*, Yasser El-Sherif, Ben Warner, Shraddha Gulati, Konstantinos Sarras, Ellie Alberts, Joe Segal, Tamir Rashid, Phil Harrison, John Devlin, Michael Heneghan, Alberto Fueyo, Gwion Emlyn, Hadil Abu Arqoub, Patrick Dubois, Deepak Joshi, Nick Powell, Bu' Hayee. King's Health Partners, London, UK

\subsection{6/gutjnl-2018-BSGAbstracts. 120}

Introduction Primary sclerosing cholangitis (PSC) is a chronic inflammatory condition of the bile ducts leading to fibrosis and end stage liver disease. A lack of robust non-invasive biomarkers has been hindering disease monitoring and development of optimal therapies. We have previously noted that the high levels of faecal calprotectin (fcal) seen in PSC-IBD patients belie the mild or quiescent intestinal inflammation. An unsupervised proteomics study identified biliary calprotectin as a potential biomarker. Here, we test the hypothesis that fcal is a marker of biliary injury in PSC.

Methods We analysed paired endoscopic activity data (UCEIS) and fcal results of patients with PSC-IBD $(n=20)$ or UC $(n=20)$ who underwent colitis surveillance in the context of a colitis surveillance pilot study. Relevant clinical data was recorded prospectively. Recruiting consecutive patients attending for ERCP $(n=6)$ allowed for the concomitant testing of biliary and faecal calprotectin.

Results As expected, fcal strongly correlated with severity of mucosal injury (UCEIS) in UC $[\mathrm{r}=0.82,95 \% \mathrm{CI}(0.58,0.92)$, $\mathrm{p}<0.0001]$. However, the correlation was weaker in PSC-IBD $[\mathrm{r}=0.59,95 \% \mathrm{CI}(0.19,0.82), \mathrm{p}=0.006]$. Moreover, in patients with PSC-IBD and quiescent colitis (UCEIS: 0-1) fcal concentration was significantly higher in comparison to UC patients with comparable endoscopic activity $[279 \mathrm{ug} / \mathrm{g}(10,1560)$ vs. $30(10,161), p=0.015)]$. A trend towards abnormal liver biochemistry was seen in those PSC-IBD with higher fcal [ALP: 250IU/L $(113,561)$ vs. $83(59,170), \mathrm{p}=0.06$, GGT: $351 \mathrm{U} / \mathrm{L}$
(117, 1014) vs. $51(29,153) \mathrm{p}=0.02$, AST: $53 \mathrm{U} / \mathrm{L}(26,85)$ vs. $37(22,43), \mathrm{p}=\mathrm{ns}$. UC patients with quiescent colitis and fcal $>150$ had a higher risk of colitis relapse in 12 months $[\mathrm{HR}=7.6,95 \% \mathrm{CI}(1.8,33.6)]$ in comparison to those with fcal $<150$. However, in patients with PSC-IBD and quiescent colitis a fcal $>150$ was associated instead with a higher risk of cholangitis associated complications (need for antibiotics or stent insertion), $\mathrm{HR}=6.5,95 \% \mathrm{CI}(1.3,33.9)$. Strikingly, biliary calprotectin concentration showed a strong correlation with fcal concentration $(r=0.90, p=0.04)$. Interestingly, immunostaining of biliary brushings for calprotectin demonstrated positive staining in cholangiocytes as well as neutrophils and macrophages.

Conclusion In patients with PSC-IBD and quiescent colitis the identification of a raised fcal is likely to herald complications of inflammation in the bile ducts rather than the colon. In this setting, fcal may be a valuable prognostic biomarker of cholangitis. Additionally, our data suggest that in PSC, the source of raised fcal may also be the damaged biliary epithelium.

\section{ADTU-05 GUT MICROBIAL COMPOSITION IN THE MIGRANT SOUTH-ASIAN IBD POPULATION IN UK}

\footnotetext{
${ }^{1,2}$ Mohammed Quraishi* ${ }^{3}{ }^{3}$ Animesh Acharjee, ${ }^{2,3}$ Neeraj Bhala, ${ }^{2}$ Shrikanth Pathmakanthan, ${ }^{2}$ Rachel Cooney, ${ }^{2,3}$ Subrata Ghosh, ${ }^{3}$ Georgios Gkoutos, ${ }^{1,2}$ Andrew Beggs, ${ }^{4}$ Amanda Rossiter*, 1,2,3 Tariq Iqbal*. ${ }^{1}$ Institute of Cancer and Genomic Sciences, University of Birmingham, Birmingham, UK; ${ }^{2}$ University Hospital Birmingham, Birmingham, UK; ${ }^{3}$ Institute of Translational Medicine, Birmingham, UK; ${ }^{4}$ Institute of Microbiology and Infection, University of Birmingham, Birmingham, UK
}

\subsection{6/gutjnl-2018-BSGAbstracts. 121}

Introduction Epidemiological studies have highlighted that the South Asian migrant population in UK have a comparable risk of developing IBD but with a more aggressive phenotype than the white Caucasian population. It remains unclear if this is due to environmental/lifestyle factors or differences in host genetics. The human gut microbiota is impacted by health status and diet and therefore represents a potentially adaptive phenotype that is influenced by the environment. As the gut microbiota has been shown to be different in the native South Asian population compared to those in developed countries, we aimed to investigate if there were ethnic differences in the microbiota in IBD patients.

Methods Stool samples were collected from South Asian $(n=20)$ and Caucasian patients $(n=46)$ with IBD attending outpatient clinics at University Hospital Birmingham along with healthy controls $(n=17)$. DNA was extracted and the V4 hyper-variable region of the $16 \mathrm{~S}$ rRNA gene amplified and sequenced. Analysis was performed on the QIIME pipeline using the GreenGenes database. Diversity analysis was corrected for false discovery rates.

Result Patients with IBD had a significantly different gut microbial composition in comparison to healthy controls as expected $(p=0.01)$. Gut microbial diversity was reduced in IBD $(p=0.001)$. A significant decrease in Firmicutes phylum was observed in patients with IBD in comparison to healthy controls which was primarily due a reduced abundance of communities from genus Faecalibacterium praunitizii, Lachnospiracae, Ruminococcus and Blautia $(\mathrm{p}<0.002)$. Within the IBD cohort, the alpha or beta diversity of gut microbiota was very similar for South Asians and Caucasian patients. No significant differences were seen at any taxonomic levels. 